\title{
Dolor Abdominal Crónico en pediatría
}

\author{
LORENA RODRÍGUEZ G. ${ }^{1}$, ROSSANA FAÚNDEZ H. ${ }^{2}$, DANIELA MAURE O. ${ }^{3}$ \\ 1. Pediatra, Gastroenteróloga Infantil. Hospital San Juan de Dios de Santiago. Clínica Alemana de Santiago. \\ 2. Pediatra,Gastroenteróloga Infantil. Hospital San Juan de Dios de Santiago. Universidad de Chile. \\ 3. Pediatra. Becada Gastroenterología Infantil. Universidad de Chile.
}

\begin{abstract}
\section{Chronic Abdominal Pain in children}

Chronic Abdominal Pain (CAP) in children is a common cause to seek medical care. This paper summarizes current physiological and biochemical concepts that help in understanding the symptomatology. A review of the main etiological causes connected to CAP is offered, as well as a systematic process to evaluate and diagnose each. Recommendations for management and referral are provided.
\end{abstract}

(Key words: Abdominal Pain, gastrointestinal disorders, Helicobacter pilory, dispepsia).

Rev Chil Pediatr 2012; 83 (3): 279-289

\section{RESUMEN}

El dolor abdominal crónico en niños es una causa común de consulta médica. El presente trabajo resume los conceptos fisiológicos y bioquímicos actuales que apuntan hacia el cabal entendimiento de la sintomatología. En este artículo se presenta una revisión de las principales causales etiológicas asociadas al dolor abdominal crónico, como asimismo un proceso sistemático para evaluar y diagnosticar cada caso. Igualmente, se proporcionan recomendaciones para su manejo y derivación a especialista.

(Palabras clave: Dolor abdominal, alteraciones gastrointestinales, Helicobacter pylory dispepsia).

Rev Chil Pediatr 2012; 83 (3): 279-289

\section{Introducción}

El dolor abdominal crónico (DAC) es una causa frecuente de consulta en pediatría general (2-4\%) y gastroenterología infantil, en particular en adolescentes (7-25\%). Se estima que $13-17 \%$ de población en edad escolar lo presenta y sobre los 9 años es más frecuente en las mujeres (5:1). Su enfrentamiento constituye un desafío constante para pediatras y gastroenterólogos infantiles Corresponde a un grupo heterogéneo de pacientes, con gran variedad de síntomas, que afecta la calidad de vida de quienes lo padecen. En los niños el dolor habitualmente es funcional $(90 \%)$ sin anormalidades fi siológicas, estructurales o bioquímicas. Según los nuevos criterios de Roma III, el DAC se define como dolor abdominal intermitente o

Trabajo recibido el 28 de diciembre de 2011, aceptado para publicación el 04 de febrero de 2012.

Correspondencia a:

Lorena Rodríguez González

E-mail: lolyrodriguez@vtr.net, Irodriguez@alemana.cl 
constante, de al menos 2 meses de duración, funcional u orgánico (por enfermedad específi ca). En esta revisión se analizan los nuevos criterios, su clasificación, evaluación clínica y enfoque terapéutico de acuerdo a las últimas publicaciones.

Apley y Naish introdujeron en 1958, el término dolor abdominal recurrente ${ }^{1}$, describiéndolo como al menos "tres o más episodios de dolor abdominal severo que interfieren con la actividad normal del niño durante un periodo mayor de tres meses". Este concepto actualmente es considerado demasiado amplio y confundente.

La Academia Americana de Pediatría (AAP) en el año 2005 y la Sociedad Norteamericana de Gastroenterología Pediátrica, Hepatología y Nutrición (NASPGHN), recomiendan retirar los términos: recurrente y recidivante. La Sociedad Americana de Dolor (APS) y la Asociación Americana de Medicina Familiar (AAFP) también adoptaron la denominación de DAC, como una descripción, que no representa una enfermedad y que agrupa multiples problemas clínicos ${ }^{2,3,4}$.

Para estandarizar los diagnósticos de los desórdenes gastrointestinales funcionales se crearon los Criterios de Roma II, posteriormente redefinidos por Roma III para niños entre 4 y 18 años, en el año 2006, como una forma de excluir enfermedades serias, disminuir el disconfort y lograr una vida normal ${ }^{5}$. Uno de los cambios importantes fue disminuir de tres a dos meses los síntomas de dolor abdominal para considerarlo crónico.

En Chile, no existen estudios de prevalencia, sin embargo, se estima que DAC explica el $13 \%$ aproximadamente de las consultas gastroenterológicas pediátricas.

\section{Clasificación del dolor abdominal crónico (DAC)}

\section{DAC órganico}

La causa orgánica debe considerarse siempre en primer lugar en menor es de 7 años, en especial de 3-4 años. La presencia de sintomas de alarma constituyen una indicación para realizar pruebas de laboratorio diagnósticas que deben solicitarse en forma escalonada ${ }^{9}$ (tabla 1 ).
Tabla 1. Principales enfermedades orgánicas relacionadas con DAC

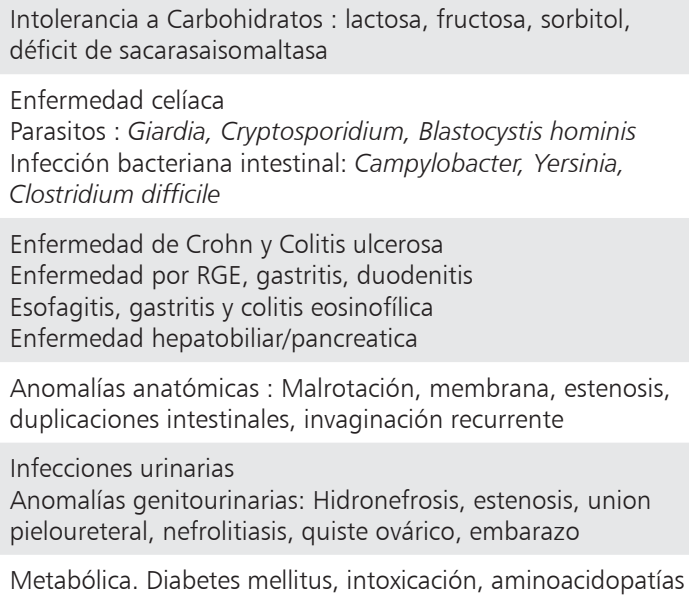

Anomalías anatómicas : Malrotación, membrana, estenosis, duplicaciones intestinales, invaginación recurrente

Infecciones urinarias

Anomalías genitourinarias: Hidronefrosis, estenosis, union

pieloureteral, nefrolitiasis, quiste ovárico, embarazo

Metabólica. Diabetes mellitus, intoxicación, aminoacidopatías

\section{DAC funcional (criterios de Roma III)}

Una vez descartada la enfermedad orgánica (ausencia de signos de alarma) se puede establecer el dianóstico de DAC funcional. Según los criterios actuales, basados en en los criterios Roma III ${ }^{6,18}$ para enfermedades funcionales gastrointestinales en niños y adolescentes, se clasifica en:

1. Dispepsia funcional.

2. Sindrome de colon irritable.

3. Migraña abdominal.

4. Dolor abdominal funcional y sindrome de dolor abdominal funcional.

\section{Historia clínica en diagnóstico diferencial de DAC}

1. Historia dirigida y examen físico completo.

2. Intentar clasificar con la historia en alguno de los patrones clínicos conocidos.

3. Antecedentes dietéticos: dolor relacionado a alimentos ofensores (por ej: lactosa, fructosa, sorbitol).

4. Antecedentes medicamentosos: identificar aquellos que exacerban o alivian dolor (AINES, bloqueadores de receptores de histamina, inhibidores de bomba de protones, etc.).

5. Historia familiar de DAR funcional o orgánico (como colon irritable, enfermedad inflamatoria intestinal, enfermedad ulcero péptica, etc.). 
6. Sobre todo en adolescentes con DAR es fundamental una historia social pertinente (en búsqueda de embarazo, enfermedades de trasmisión sexual, procesos inflamatorios pélvicos, etc.).

\section{Signos y síntomas de alarma en DAC}

Existen ciertos factores de riesgo independientes, llamados "síntomas de alarma" o "banderas rojas", ya que incrementan la probabilidad de etiología orgánica subyacente y disminuyen la probabilidad de diagnóstico funcional.

1. Edad: menor a 4 años.

2. Dolor: localizado, excéntrico, irradiado a dorso-hombro. Dolor persistente en cuadrantes superior e inferior derecho, que despierta en la noche. Distensión o efecto de masa, hepato esplenomegalia.

3. Palpación costo vertebral dolorosa, lesiones peri anales (úlceras perirectales y/o fisuras anales).

4. Pérdida de peso involuntaria, retraso de pubertad, retardo del crecimiento.

5. Diarrea nocturna, sangrado gastrointestinal, hemorragia oculta.

6. Estado nauseoso, vómitos. Disfagia.

7. Síntomas extra intestinales: fiebre de causa no explicable.

8. Rush, uveítis, artritis, artralgia.

9. Disuria.

10. Historia familiar de enfermedad inflamatoria intestinal, celiaca o ulcerosa.

11. Antecedente de inmunocompromiso congénito o adquirido.

12. Uso de medicamentos antiinflamatorios no esteroidales, cortocoesterides, ácido acetil salicílico.

13.Laboratorio básico anormal: anemia, VHS elevada, parásitos en deposiciones

14. Historia de viaje al exterior, exposición a agua o leche contaminada.

\section{Una correcta estimación del dolor incluye}

1. Localización. La del dolor funcional con frecuencia es peri umbilical o difusa; la del dolor orgánico es más precisa, dependiendo del órgano afectado, o la enfermedad en sí misma.

2. Irradiación.
3. Periodicidad. La del dolor originado en vísceras, transmitido por vía del SNA, es generalmente intermitente, mientras que la del dolor de cualquier origen, trasmitido por vía somática (nervios espinales) es generalmente de carácter continuo.

4. Ritmo, horario. Ritmo diurno: no tiene gran significado en ausencia de una relación digestiva evidente. Aparición nocturna: sugiere enfermedad orgánica.

5. Relación con la ingestión de alimentos: postprandial precoz, tardío, o sin relación prandial aparente.

6. Intensidad.

7. Calidad y carácter (cómo sienten el dolor): quemante ardoroso, penetrante, lancinante, constrictivo, sordo o pulsátil; calambre, pesantez, etc.

8. Modo de comienzo y de alivio.

9. Variación con los cambios de posición.

10. Síntomas asociados existentes.

11. Curso. Regular: generalmente asociados a

12.Afección orgánica. Irregular: más frecuentes en procesos funcionales.

\section{Estudio complementario inicial}

1. Laboratorio: Hemograma. VHS. Perfil Bioquímico (ALT, AST, GGT y albúmina, amilasa y lipasa).

2. Deposiciones: Estudio parasitológico seriado en heces, Elisa para Giardia. Hemorragias ocultas en deposiciones, leucocitos eosinófilos.

3. Orina Completa y urocultivo.

4. Ecografía abdominal y pélvica. Se debe reservar estas pruebas para:

- Aquellos que cumplen con síntomas de alarma" o "aquellos con síntomas específicos que sugieran enfermedad orgánica".

- Para la seguridad de paciente-padre-médico de la ausencia de organicidad, particularmente si dolor disminuye significativamente la calidad de vida del paciente.

\section{Estudio complementario específico}

- Serología de Enfermedad celíaca. Endoscopia, biopsia intestinal, estudio Helicobacter (H) Pylori, test hidrogeno espirado. Trán- 
sito intestinal, enema baritado, manometría anorectal (EMAR), colonoscopia, TAC, cápsula endoscópica y otras exploraciones.

- De las pruebas de imagen, en ausencia de síntomas de alarma es discutible incluso el empleo sistemático de la radiografía de abdomen simple y de la ecografía abdominal (hallazgos en $<1 \%$ ).

- Cuando síntomas atípicos están presentes como ictericia, molestias urinarias, dolor en flancos o fosas iliacas, la ecografía es más probable que detecte anormalidades (10\%).

- Otras pruebas invasivas, como endoscopías, sólo estarían indicadas en sospecha clara de organicidad.

- En un porcentaje elevado de casos la endoscopía alta y baja (colonoscopía) diagnostica anomalías histológicas inflamatorias de tubo digestivo alto y bajo, sin que necesariamente ello implique una función patogénica de la inflamación de la mucosa gastrointestinal en el DAR.

- Es difícil trazar un esquema general de asistencia para todos los pacientes con DAC, porque la atención debe ser muy individualizada de acuerdo a las características particulares de cada caso. Una guía útil siempre parte de una correcta anamnesis y examen físico exhaustivo, de utilidad también para determinar si el paciente requiere o no hospitalización para continuar su estudio, si puede evaluarse en una consulta general, o si debe remitirse a una consulta especializada.

\section{Dolor abdominal crónico funcional (DACF)}

Es la causa más frecuente de dolor abdominal crónico. Forma parte de los desórdenes gastrointestinales funcionales del niño y adolescente, definidos por Roma II y redefinidos por los criterios Roma III , como "a lo menos 8 semanas no consecutivas de dolor abdominal que interfiere la actividad normal, sin relación o relación muy ocasional con eventos fisiológicos".

\section{Fisiopatología}

En su génesis intervienen 2 constantes fisiopatológicas: "hipersensibilidad visceral" y “dismotilidad intestinal” (figura 1).
Los niños con DACF tendrían disminuido el umbral doloroso visceral y responderían frente a estímulos convencionales de forma exacerbada (hiperalgesia), y así el gas generado en la digestión alimenticia o el efecto presor del bolo o cambios sutiles en su composición, que por lo habitual no son estímulos dolorosos, actuarían como desencadenantes. Además la dismotilidad gastrointestinal y cólica ha sido demostrada en numerosos estudios manométricos.

Las alteraciones de la motilidad podrían ser resultado de una disautonomía vegetativa, y más específicamente de su actividad simpática aferente en el tracto gastrointestinal, originando calambres por peristaltismo anómalo exacerbado.

Se piensa que los síntomas pueden surgir a partir de cambios en el "cerebro del intestino" (ej. Gastroenteritis aguda), constituido por las neuronas de los plexos intestinales con función conectada al sistema nervioso central (SNC), que controlan la motilidad, la secreción y participan en la regulación de procesos inmunes e inflamatorios a través de neurotransmisores. El sistema nervioso entérico (SNE) está conectado al Sistema neural autonómico y al SNC a través de vías sensitivas y motoras del $\mathrm{SN}$ Simpático y Parasimpático. Los estímulos que inician esta reactividad anormal pueden ser neurógenos (comida, distensión del intestino, cambios hormonales), orgánicos (procesos inflamatorios) o psicológicos (separación de los padres, ansiedad).

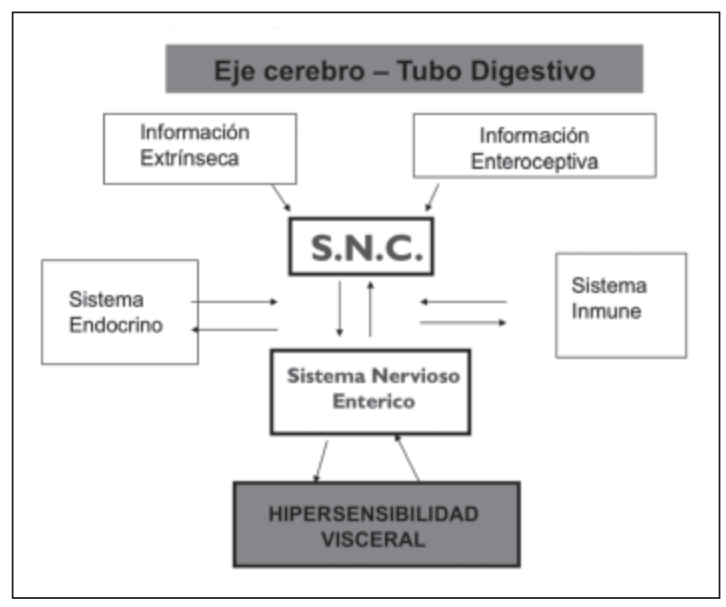

Figura 1. 
El camino a la cronicidad del dolor se caracteriza por intentos fallidos de ajustarse y enfrentarse a experiencias adversas. Para que el dolor se haga crónico, es preciso el concurso de estímulos estresantes físicos (medicación reciente, estreñimiento, aerofagia, intolerancia la lactosa, etc.) o psíquicos (muerte, divorcio de los padres, enfermedad, acoso escolar por otros niños, etc.). Con el transcurso del tiempo, la reiteración de estas experiencias conduce al paciente a desarrollar síntomas concomitantes de incapacidad física crónica, ansiedad, trastornos del sueño, ausencias escolares y retirada social.

No obstante para que el dolor abdominal se haga recurrente o crónico es preciso el concurso de estímulos estresantes tanto de orden físico como psíquico, como "familias dolientes", "niños víctimas", "niños abusados", "fobias escolares", "expectativas paternas excesivas", todas ellas asociadas al modelo conceptual biopsicosocial de enfermedad (figura 2).

\section{Modelo conceptual biopsicosocial}

El horario escolar a veces es intenso y con elevadas exigencias, como respuesta a un mundo cada vez más competitivo, lo que fuer- za al niño a cumplir expectativas paternas excesivas. Los niños tienden a ser introvertidos, con escasa autoestima, ansiedad, aislamiento y depresión, con dificultad para reaccionar adecuadamente al enfrentarse a problemas rutinarios que no pueden superar.

El dolor abdominal crónico funcional forma parte de los desórdenes gastrointestinales funcionales de niños y adolescentes (tabla 2).

Los trastornos funcionales pediátricos relacionados con dolor abdominal y sus características, siguiendo los criterios de Roma III son: (deben cumplirse al menos una vez a la semana al menos 2 meses antes de diagnóstico).

\section{Dispepsia funcional (incluye todos ellos)}

"Dolor o molestia epigástrica postprandial o no, asociada a náuseas y vómitos, o saciedad y plenitud gástrica".

\section{Criterios diagnósticos}

1. Dolor persistente o recurrente ubicado en abdomen superior (sobre ombligo).

2. No mejora con la defecación, no se asocia con cambios en la frecuencia o forma de deposiciones (no colon irritable).

3. No hay evidencia de proceso inflamatorio, anatómico, metabólico o neoplásico que explique síntomas.

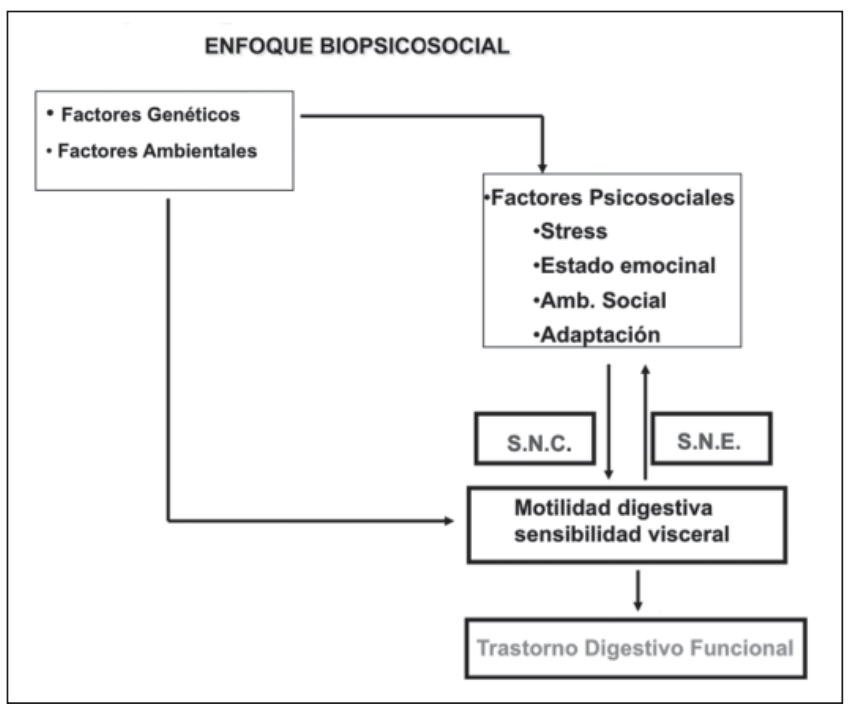

Figura 2. SNC: Sistema nervioso central. SNE: Sistema nervioso entérico.
Tabla 2.

H1. Vómitos y aerofagia
H1a. Síndrome de rumiación del adolescente
H1b. Síndrome de vómitos cíclicos
H1c. Aerofagia
H2. Desórdenes Gl funcionales asociados a dolor
abdominal
H2a. Dispepsia funcional
H2b. Síndrome de intestino irritable
H2c. Migraña abdominal
H2d. Dolor abdominal funcional del niño
H2d1. Síndrome de dolor abdominal
funcional del niño
H3. Constipación y Incontinencia
H3a. Constipación funcional
H3b. Incontinencia fecal no retentiva


Su diagnóstico diferencial incluye dispepsia funcional (sin causa orgánica) y dispepsia orgánica, o secundaria a: inflamación de tubo digestivo superior, desórdenes de motilidad o enfermedades gastrointestinales.

Puede adoptar 2 patrones clínicos: dispepsia tipo péptica o tipo ulcerosa (regurgitación, disfagia, pirosis, náuseas, vómitos) y dispepsia tipo dismotilidad (saciedad precoz, hipo excesivo, eructos, arcadas).

\section{Síndrome de colon irritable}

"Dolor tipo cólico, difuso, periódico, que cursa con alteraciones del ritmo intestinal (diarrea, estreñimiento) debe incluir los siguientes criterios diagnósticos:

1. Dolor abdominal o molestia abdominal asociado a dos o más de lo siguientes aspectos, al menos el $25 \%$ del tiempo:

Se alivia o mejora con la defecación.

Se asocia con cambios en frecuencia de deposiciones.

Se asocia con cambio en la consistencia de las deposiciones (más duras o líquidas).

2. Con ausencia de alteraciones estructurales o bioquímicas que expliquen los síntomas.

Puede subclasificarse en: "con constipación predominante" o "con diarrea predominante".

Su diagnóstico diferencial incluye: enfermedades inflamatorias intestinales, infecciones y parasitosis intestinales, intolerancia a lactosa y otros azúcares, síndromes de malabsorción, diarreas y estreñimientos farmacológicos, trastornos ginecológicos, neoplasias.

\section{Migraña abdominal}

Al menos 3 episodios paroxísticos de intenso dolor abdominal dentro de los últimos 12 meses, de duración desde 2 horas a varios días, con intervalos libres de síntomas desde semanas a meses.

Para el diagnóstico se requiere:

1. Ausencia de evidencias de anomalías metabólicas, gastrointestinales y estructurales o bioquímicas de SNC.

2. Presencia de a lo menos 2 de las siguientes: cefalea durante los episodios, fotofobia durante los episodios, historia familiar de migraña, cefalea confinada a un solo sitio, o un aura o periodo prodrómico consistente en ya sea síntomas visuales (visión borrosa o restringida), sensoriales o motores (hablar limitado o incapacidad para hablar, etc.).

Algunos niños no cumplen con los criterios clásicos, pero igual responden a la terapia antimigrañosa ("familias dolientes", "niños víctimas", "niños abusados", "fobias escolares", etc.).

\section{Dolor abdominal funcional y síndrome de dolor abdominal funcional}

"Episodios de dolor abdominal paroxístico periumbilical (DAPP) que se alternan con periodos libres de síntomas". Interrumpe las actividades del niño o adolescente y dificulta su escolaridad o actividad académica.

Su diagnóstico diferencial incluye desórdenes funcionales como DAC Funcional y Migraña abdominal (cuando se asocia a cefalea, náuseas, fotofobia, $\mathrm{y}$ vómitos u otros síntomas vegetativos como sudoración y palidez) y desórdenes orgánicos.

En la etiopatogenia del DAPP funcional destacan alteraciones de la conducta; como simulación, conversión y somatización.

El DAPP que no se irradia, que no lo despierta durante el sueño y no induce pérdida de peso o retraso en los parámetros de crecimiento, es típico de funcional.

\section{Principios generales del estudio diagnóstico en el dolor abdominal crónico funcional}

El dolor abdominal crónico funcional (DACF) puede ser diagnosticado por médicos de cuidado primario en niños, sin requerir evaluación diagnóstica adicional.

Si por clínica (historia compatible y examen físico normal) cumple con criterios de DAC funcional o Síndrome de intestino irritable se aconseja iniciar una terapia empírica más que iniciar una evaluación extensa.

Idealmente, el diagnóstico de funcional debe establecerse de entrada y no a través de un "ir y venir" para practicar exploraciones complementarias costosas y engorrosas.

El médico que atiende niños y adolescentes no debe alentar expectativas diagnósticas 
en los padres $u$ hospitalizar al niño o adolescente en forma innecesaria, porque reforzaría el comportamiento ambiental al dolor y perpetuaría el trastorno.

\section{Pronóstico en DAC Funcional}

El DACF parece ser una condición crónica que acompaña a lo largo de la vida y se inicia en la niñez, y su pronóstico es bueno ${ }^{7,8}$ :

- 1/3 resuelven síntomas dentro de 2 meses del diagnóstico.

- 1/3 tienen desórdenes a largo plazo como depresión, ansiedad y dolor abdominal en su adultez.

- 1/3 tiene otras quejas crónicas además de DACF como Jaqueca.

En promedio el 50\% de los niños sigue con dolor después de 3 años de seguimiento, y la aceptación del modelo biopsicosocial de enfermedad por los padres se asocia fuertemente a recuperación.

Apley encontró varios factores que predicen mal pronóstico a largo plazo: sexo masculino, inicio precoz antes de los 6 años, retraso en el diagnóstico ( $>6$ meses), y una historia familiar de dolor somático.

\section{DAC y Helicobacter pylori}

Aunque existe relación confirmada entre infección por $H$. pylori y presencia de gastritis, la asociación de la misma con el DAR es muy débil, además la mayoría de los niños infectados son asintomáticos.

Como no existe suficiente evidencia científica de que el tratamiento erradicador en Gastritis H. pylori sin úlcera sea beneficioso para la desaparición de los síntomas, no está indicada la búsqueda rutinaria de la infección por $H$. pylori mediante métodos diagnósticos no invasivos.

Si durante el estudio endoscópico se identifica $H$. pylori y el paciente presenta una gastritis sin úlcera, debe considerarse ofrecer una terapia erradicadora, explicando a la familia y al niño la insuficiencia actual de pruebas a favor o en contra de que dicho tratamiento desaparezca la sintomatología.

\section{Derivación a gastroenterólogo infantil}

- Presencia de "síntomas de alarma".

- Sospecha de causa orgánica gastrointestinal.

- Fracaso de terapia empírica (1-2 meses).

- Síntomas inexplicados por más de tres meses.

- Si requiere endoscopía alta: fracaso de terapia supresora de ácido en DAR, evidencias de laboratorio de enfermedad (anemia por déficit de fierro, VHS elevada, serología celíaca).

- Si requiere endoscopía baja o colonoscopía: sangrado rectal, evidencias de laboratorio de enfermedad (anemia por déficit de fierro, VHS elevada, serología para enfermedad inflamatoria intestinal), fracaso de terapia antiespasmódica para DAR.

\section{Tratamiento}

- Debe estar dirigido a recuperar la actividad normal y no al manejo del dolor propiamente tal, debiendo ser conversado claramente con los padres y paciente, confirmándoles que el dolor es real, aunque de naturaleza funcional, y que la malignidad es rara en niños y adolescentes.

- Es importante explicar y proporcionar ejemplos simples de hiperalgesia (como piel dañada), y de manifestaciones de interacción entre el cerebro y intestino (vómitos y diarrea frente a situaciones de stress).

- Los padres deben saber que no es una enfermedad seria, que amenace la vida y que el DAR Funcional es un diagnóstico válido y aceptado.

- Si cumple cabalmente con los criterios de funcional, puede iniciarse tratamiento empírico según patrón clínico predominante ${ }^{17,18}$.

1. Dieta rica en fibras, ablandadores de deposiciones (en Síndrome de intestino irritable (SII) de predominio constipación).

2. Procinéticos, como metoclopramida, eritromicina, domperidona (en Dispepsia tipo dismotilidad y Aerofagia).

3. Bloqueadores $\mathrm{H} 2$, inhibidores de bomba de protones (en dispepsia tipo ulcerosa). 
4. Antiespasmódicos, agentes antidiarreicos (loperamida), relajantes de musculatura lisa, anticolinérgicos (en SII de predominio diarrea).

5. Antidepresivos tricíclicos (amitriptilina), inhibidores de la recaptación de serotonina (citalopram), éstos dos últimos agentes supervisados por médico psiquiatra, en caso de transtorno psicológico concomitante.

6. Antagonistas de receptores H1 (ciproheptadina), antagonistas de receptores de serotonina (pizotifeno) y propranolol (en migraña abdominal), o sumatriptan.

Debido a que las comorbilidades psicológicas tales como ansiedad (33-77\%) y depresión $(9-40 \%)$, pueden impedir la recuperación médica y psicológica, es necesario enfrentar estos problemas con la ayuda de psicólogo y psiquiatra. La terapia conductual y cognitiva juega un importante rol en tratamiento de DAR Funcional. Los "Gatillantes ambientales de dolor" deben ser identificados y la "fobia escolar" debe ser considerada. También deben ser considerados: "estados de desadaptación", "dinámica familiar alterada", "niños o adolescentes víctimas", "niños o adolescentes abusados", etc.

\section{Tratamiento no farmacológico}

Independientemente del origen orgánico o funcional del DAC, la educación de la familia y del propio niño es un capítulo importante del tratamiento. Si se sospecha DAC funcional, hay que explicarles de forma sencilla en qué consiste y que no es una "rareza", sino algo común, así como sus manifestaciones y cómo se originan, asegurándole que no tiene un trastorno serio o crónico.

Puede requerirse la participación de psicología y psiquiatría infantil, fundamentalmente en pacientes con reacciones de conversión, ansiedad incontrolable, imitación del patrón familiar y conflictos familiares.

Deben modificarse conductas nocivas y eliminar en lo posible aquellas situaciones que generen estrés al paciente. Un programa de terapia cognitiva-conductual y una intervención familiar similar, han dado muestras de ser eficaces en el tratamiento del DAC funcional, con disminución de la intensidad y frecuencia de aparición de los episodios dolorosos.

Es necesario eliminar la fuente de las consecuencias negativas psicosociales del dolor, como las ausencias a la escuela, así como promover actividades que incrementen la independencia del niño.

El objetivo es ayudarlo a controlar y tolerar el dolor por sí mismo, mientras participa plenamente en las actividades cotidianas. Luego se trabajará con la familia para eliminar el estrés innecesario y ayudarlo a sobrellevar mejor aquel que es inevitable. Muchas veces se requiere involucrar al personal de la escuela en la terapia.

\section{Terapia psicológica}

Para enfrentar adecuadamente a estos niños se propone el "Modelo Biopsicosocial”, que propone que el DACF en el niño responde a factores biológicos, gobernados por la interacción entre el temperamento del niño, de la familia y del medio escolar.

Los síntomas del niño pueden ser influenciados por el modelo conceptual de enfermedad de los padres. Con alta frecuencia los padres necesitan encontrar "una causa" tanto como "la cura" para el dolor de sus hijos". La necesidad de encontrar "una causa más que una razón", hace difícil aceptar los factores psicológicos, en particular los desordenes relacionados a stress, como causa de dolor.

La intervención psicológica tiene un rol importante, y las metas de la terapia psicológica son modificar pensamientos, opiniones, y respuestas conductuales para síntomas y efectos de la enfermedad.

Además se cuenta con herramientas como terapias de relajación, de reforzamiento, hipnosis y terapias familiares.

Existe buena evidencia que tratamientos psicológicos, principalmente relajación y terapia conductual (aproximación usando principios de acondicionamiento) y cognitiva (aproximación en base a revisión de pensamientos y creencias), son efectivos en reducir la severidad y frecuencia de dolor crónico en el niño, sobre todo cefalea. 
El DAC es una entidad clínica frecuente y su enfrentamiento constituye un desafío para pediatras y gastroenterólogos infantiles (ver anexo 1 y 2). Su diagnóstico diferencial es extenso, pero dominado por los desórdenes funcionales. Debe mantenerse un alto índice de sospecha con el origen orgánico de dolor abdominal.

Debido a que Dolor abdominal paroxístico peri umbilical funcional (DAPPF), Dispepsia funcional y Síndrome de intestino irritable (SII) son frecuentes y definidos por criterios clínicos; esto hace actualmente prudente iniciar una terapia empírica para el desorden fun- cional sospechado mientras se concreta estudio de laboratorio, limitado para excluir causas orgánicas.

Los signos y síntomas de alarma ayudan a identificar a niños con riesgo mayor de causa orgánica específica subyacente y estrechan el enfoque de evaluación diagnóstica.

No es posible aplicar un tratamiento específico pues no existe una clara evidencia de su beneficio. Debido a la frecuencia de dinámica familiar alterada, a interacción con comorbilidad psicológica, y a estados de desadaptación en el niño, el rol del psicólogo es crítico en muchos casos.

Anexo 1

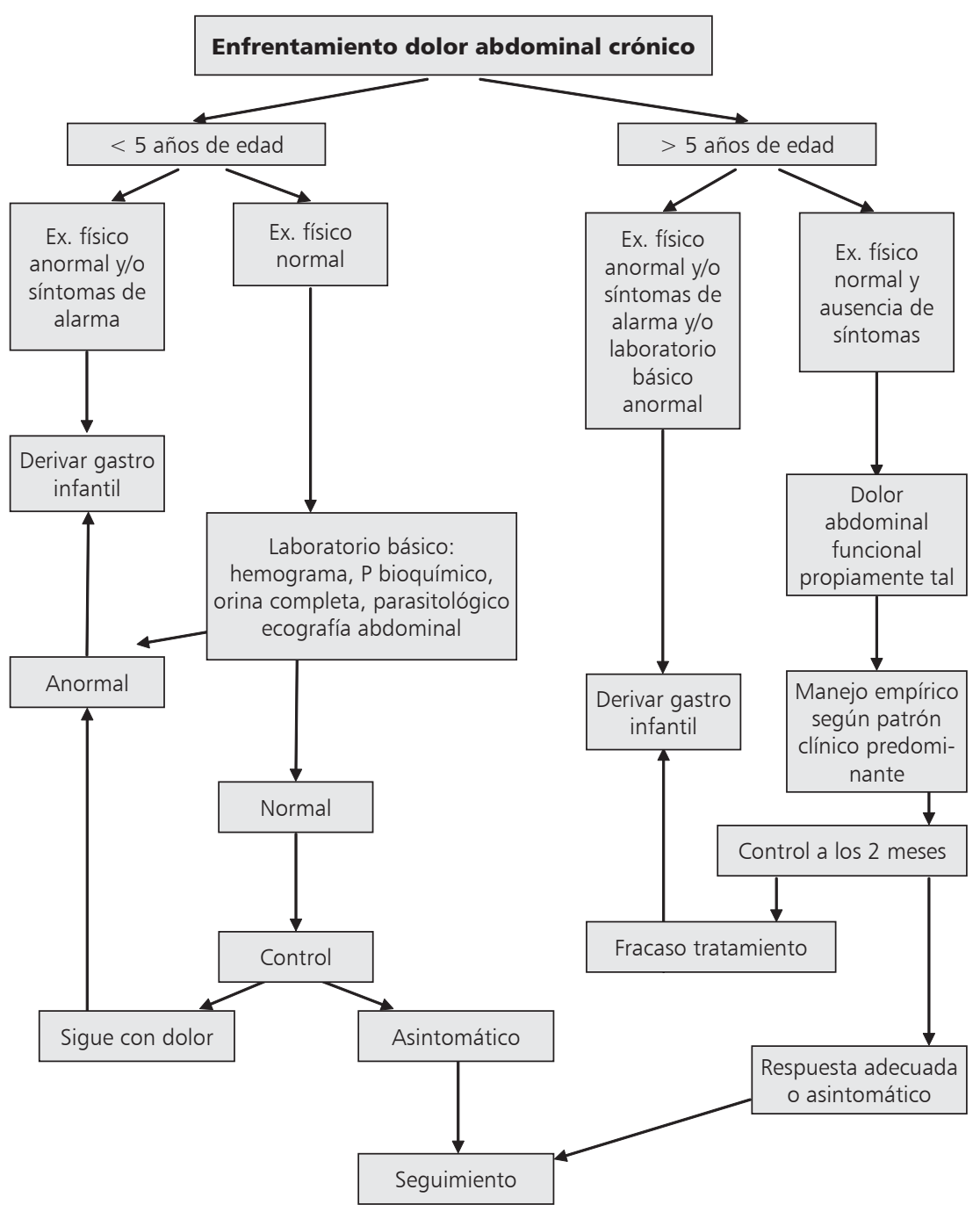


Anexo 2. Manejo de dolor abdominal crónico funcional

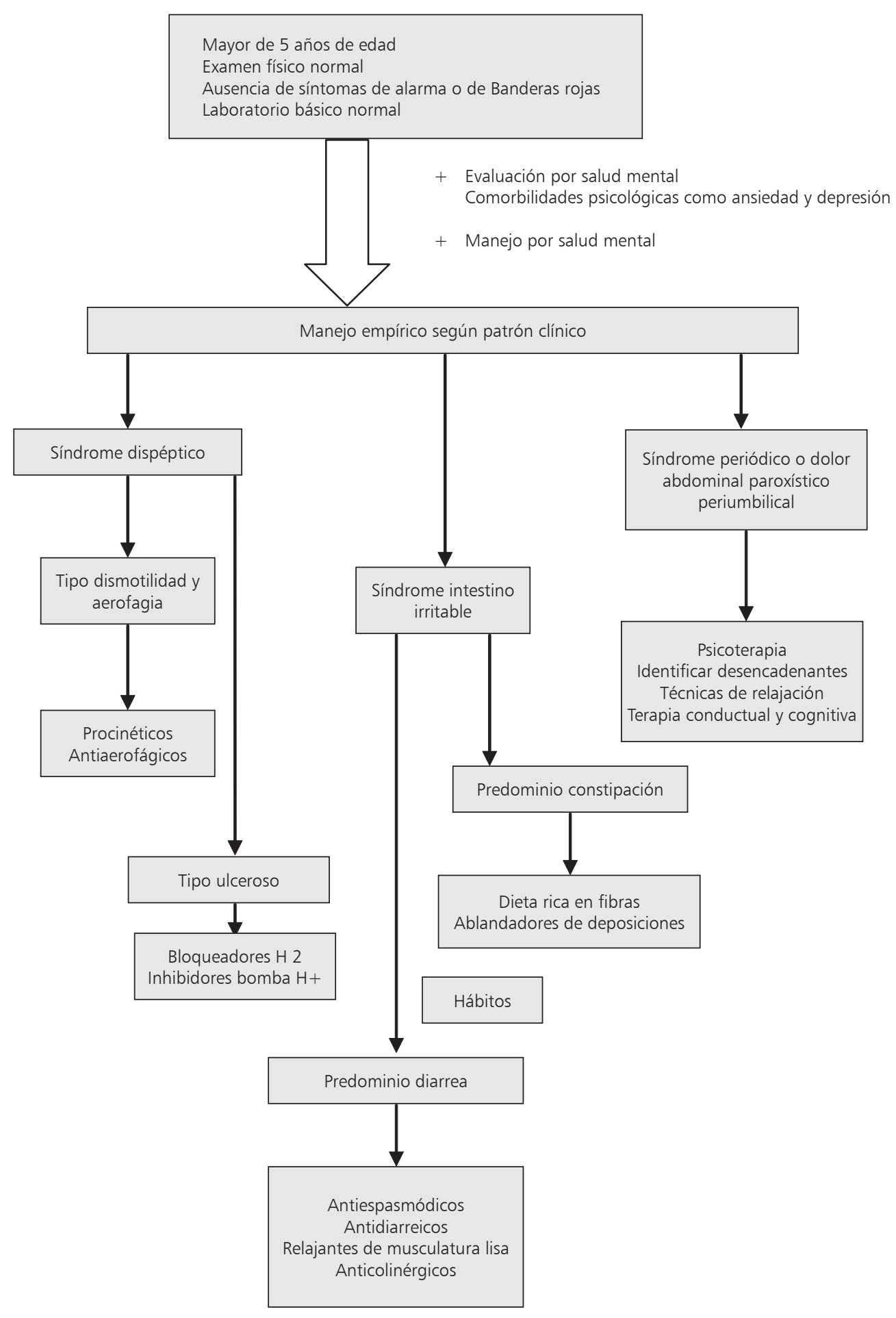




\section{Referencias}

1.- Appley J, Naish $N$ : Recurrent abdominal pain: a field survey of 1000 school children. Arch Dis Child 1958; 33: $165-70$.

2.- AAP Subcommittee and NASPGHAN Committee on Chronic abdominal pain. Chronic abdominal pain in children: A technical report of the American Academy of Pediatrics and the North American Society for Pediatric Gastroenterology, Hepatology and Nutrition. J Pediatr Gastroenter Nutr 2005; 40: 249-61.

3.- Di Lorenzo C, Colleti RB, et al: A technical report of the American Academy of Pediatrics and the North American Society for Pediatric Gastroenterology, Hepatology and Nutrition. J Pediatr Gastroenter Nutr 2005; 40: 245 8.

4.- American Academy of Pediatrics Subcommittee on Chronic Abdominal Pain; North American Society for Pediatric Gastroenterology, Hepatology and Nutrition. Pediatrics 2005; 115: 812-5.

5.- Drossman D: The Functional gastrointestinal disorders $\&$ the Rome III process. Gastroenterology 2006; 130 (5): 1377-90.

6.- Baber KF, Anderson J, et al: Rome II versus Rome III classificaction of functional gastrointestinal disorders in pediatric chronic abdominal pain. J Pediatr Gastroenter Nutr 2008; 47: 299-302.

7.- Gieteling MJ, Bierma Z et al: Prognosis of chronic or recurrent abdominal pain in children. J Pediatr Gastroenter Nutr 2008; 47: 316-26.

8.- Ramchandani P, et al: The epidemiology of Recurrent Pain in Western Countries: A systematic review. Am J
Gastroenterol 2005; 100: 1868-75.

9.- Kholi R, Li BU: Differential diagnosis of recurrent abdominal pain: new considerations. Pediatr Ann 2004 Feb; 33(2): 113-22.

10.- Plunkett A, Beattie RM: Recurrent abdominal pain in childhood. Journal Royal Society of Med 2005 Mar; 98(3): 101-8.

11.- Walker L, Lipani T: Recurrent abdominal pain: symptom subtypes based of the Rome II criteria for pediatric functional gastrointestinal disorders. Journal of Pediatric Gastroenterology and Nutrition 2004 Feb, 38(2): 18791.

12.- Urruzuno P, Bousoño C: Dolor abdominal. An Esp Pediatr 2002; 565: 452-8.

13.- García MD, García JI: Guías prácticas sobre nutrición (VII), Trastornos intestinales funcionales (equivalentes al colon irritable). An Esp Pediatr 2002; 57(3): 253-63.

14.- Rana Fayez Ammoury: Functional gastrointestinal disorders: past and present. World J Pediatr 2009; 5(2): 103-12.

15.- Rasquin A, Di Lorenzo C, Forbes D, Guiraldes E: Childhood Functional Gastrointestinal Disorders: Child/ Adolescent. Gastroenterology 2006; 130: 1527-37.

16.- González López SL: Dolor abdominal crónico en niños: conducta en la consulta médica comunitaria. Revista Electrónica de las Ciencias Médicas en Cienfuegos. Medisur 2007; 5(1): 48-55.

17.- Pereda A, Berbel O, Maluenda C: Dolor abdominal Crónico y recurrente en el niño y a dolescente. En Tratamiento en Gastroenterología, Hepatología y Nutrición pediátrica, 2a Edición. Madrid: Ergón; 2008.p 41-50.

18.- Up to Date 2011: Chronic abdominal pain in children. 